\title{
WORKPIECE PLACEMENT OPTIMIZATION OF SIX-REVOLUTE INDUSTRIAL SERIAL ROBOTS FOR MACHINING OPERATIONS
}

\author{
Claire Dumas, Stéphane Caro, Sébastien Garnier, Benoît Furet \\ Institut de Recherche en Communications et Cybernétique de Nantes (IRCCyN) \\ UMR CNRS n 6597 \\ 1, rue de la Noë, 44321 Nantes Cedex 03, France \\ Emails: $\{$ claire.dumas, stephane.caro, sebastien.garnier, benoit.furet\}@irccyn.ec-nantes.fr
}

\begin{abstract}
Roboticists are faced with new challenges in robotic-based manufacturing. Up to now manufacturing operations that require both high stiffness and accuracy have been mainly realized by using computer numerical control machine tools. This paper aims to show that manufacturing finishing tasks can be performed with robotic cells knowing the process cutting phenomena and the robot stiffness throughout its Cartesian workspace.

It makes sense that the finishing task of large parts would be cheaper with robots. However, machining robots have not been adapted for such operations yet. As a consequence, this paper introduces a methodology that aims to determine the best placement of the workpiece to be machined knowing the cutting forces exerted on the tool and the elastostatic model of the robot. In this vein, a machining quality criterion is proposed and an optimization problem is formulated. The KUKA KR270-2 robot is used as an illustrative example throughout the paper.
\end{abstract}

\section{NOMENCLATURE}

$a_{p} \quad$ Depth of cut

$f_{z}$ Feed per tooth

$F_{t} \quad$ Tangential component of the cutting force

$F_{r} \quad$ Radial component of the cutting force

$N$ Rotational speed of the tool

$K_{t}$ Coefficient for tangential component of the cutting force

$K_{r} \quad$ Coefficient for radial component of the cutting force

$V_{f} \quad$ Feed speed

\section{INTRODUCTION}

Industrial robots were originally dedicated to "pick and place" operations. They start to be used for machining operations such as trimming, deflashing, degating, sanding and sawing. Machining robots are currently developed either to help the operator realize machining operations, which have been performed with Computer Numerical Control (CNC) machines so far. First, industrial robots can be used to reduce scrap rates and production cost and to increase the volume and the flexibility of production lines. The second case deals with some applications that require $\mathrm{CNC}$ machine tools because of the expected machining quality. As a matter of fact, industrial robots are not as accurate as CNC machine tools. They are three to four times cheaper than their counterparts for a given workspace. Therefore, it makes sense that the larger the parts to be machined, the more interesting robot machining.

Some research works on robotic-based manufacturing have been conducted the last two decades. Those research works also considered parallel robots [1], which turn to have a smaller footprint ratio than industrial six-axis robots. The larger the part to be machined, the better the robots with large footprint ratio. The main drawback of industrial serial robots for machining operations turns to be their lack of stiffness and as a result, the large manufacturing errors [2, 3, 4, 5]. Indeed, a conventional machine tool is about fifty times stiffer than an industrial serial robot [3].

Matsuoka et al. [2] studied the robotic-based milling. They claimed that the machining quality can be improved by decreasing the cutting forces, namely, by decreasing the diameter of the tool and by increasing the spindle speed. Zhang et al. [3] 
proposed a real-time compensation of the robot end-effector displacement, based on the evaluation of each joint deflection. To this end, they developed an elastostatic model of the robot at hand and used a wrench sensor to measure the forces and moments applied on the robot end-effector at any time. Besides, Olabi et al. [5] showed that the robot manufacturing errors can be reduced with jerk-limited trajectories.

The vibration of the robot itself is another issue to deal with in robotic machining [4,6]. As a matter of fact, the first natural frequency of an industrial robot is about 10 to 100 times smaller than the one of conventional machine tools [6]. Pan et al. also noticed that the vibrations occurring during robotic machining operations are not regenerative but structural [6]. Therefore, they came up with a stability criterion from the elastodynamic modeling of the robot and the cutting forces. Then, they formulated some rules for robotic machining from several experimental tests. The placement of the workpiece to be machined may also affect its machining quality and/or the robot energy consumption. In this vein, Ur-Rehman et al. focused on the multiobjective path placement optimization of a parallel kinematics machines based on energy consumption, shaking forces and maximum actuators torques [7].

It makes sense that the finishing task of large parts would be more reliable and cheaper with robots. However, machining robots have not been adapted for such operations yet. As a consequence, this paper introduces a methodology that aims to determine the best placement of the workpiece to be machined knowing the cutting forces exerted on the tool and the elastostatic model of the robot. In this vein, a machining quality criterion is proposed and an optimization problem is formulated. The KUKA KR270-2 robot is used as an illustrative example throughout the paper.

The paper is organized as follows: Section 2 presents the model used to predict the cutting forces applied by the tool on the workpiece. Section 3 deals with the joint stiffness identification of the KUKA KR270-2 robot. Section 4 shows the influence of the workpiece placement and the kinematic redundancy on the machining quality of a test workpiece through some experimental results. Section 5 introduces a methodology that aims to determine the best placement of the workpiece to be machined knowing the cutting forces exerted on the tool and the elastostatic model of the robot. The proposed methodology is explained through the formulation of a mono-objective optimization problem. Section 6 shows some results of the optimization of the optimization problem at hand. Section 7 is about the conclusions of the paper and the future works.

\section{CUTTING FORCE MODEL}

The tool is composed of several teeth. We consider in this paper a tool composed of four teeth. Figure 1 presents the chip and the directions of the tangential force $F_{t}$ and the radial force
$F_{r}$ applied by each tooth on the workpiece.

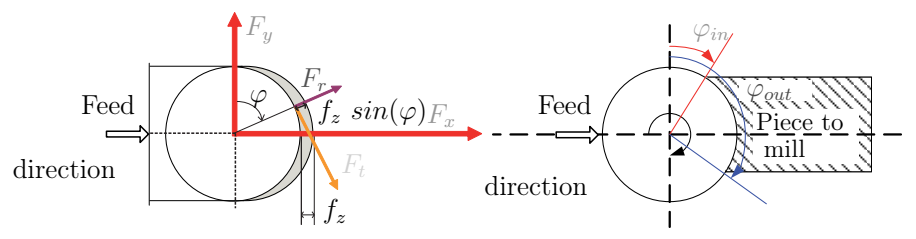

FIGURE 1. Chip geometry and definition of $\varphi_{\text {in }}$ and $\varphi_{\text {out }}$

The instantaneous forces exerted by the tooth on the workpiece are defined in [13] and are expressed as follows:

$$
\begin{aligned}
& \delta F_{t}=K_{t} a_{p} f_{z} \sin (\varphi) d \varphi \\
& \delta F_{r}=K_{r}\left\|\delta F_{t}\right\|
\end{aligned}
$$

The mean forces $F_{x}$ and $F_{y}$ along the $x$ and $y$ axes associated with one tooth are expressed as:

$$
\begin{aligned}
& F_{x}=\int_{\varphi_{\text {out }}}^{\varphi_{\text {in }}}\left(\left\|F_{t}\right\| \cos (\varphi)+\left\|F_{r}\right\| \sin (\varphi)\right) d \varphi \\
& F_{y}=\int_{\varphi_{\text {out }}}^{\varphi_{\text {in }}}\left(-\left\|F_{t}\right\| \sin (\varphi)+\left\|F_{r}\right\| \cos (\varphi)\right) d \varphi
\end{aligned}
$$

Therefore, if $\varphi \in\left[\varphi_{\text {in }} \varphi_{\text {out }}\right]$,

$$
\begin{aligned}
F_{x}= & K_{t} a_{p} f_{z}\left(-\frac{\cos \left(2 \varphi_{\text {out }}\right)}{4}+K_{r} \frac{\varphi_{\text {out }}}{2}-K_{r} \frac{\sin \left(2 \varphi_{\text {out }}\right)}{4}\right)+ \\
& K_{t} a_{p} f_{z}\left(\frac{\cos \left(2 \varphi_{\text {in }}\right)}{4}-K_{r} \frac{\varphi_{\text {in }}}{2}+K_{r} \frac{\sin \left(2 \varphi_{\text {in }}\right)}{4}\right) \\
F_{y}= & K_{t} a_{p} f_{z}\left(\frac{\sin \left(2 \varphi_{\text {out }}\right)}{4}-\frac{\varphi_{\text {out }}}{2}-K_{r} \frac{\cos \left(2 \varphi_{\text {out }}\right)}{4}\right)+ \\
& K_{t} a_{p} f_{z}\left(-\frac{\sin \left(2 \varphi_{\text {in }}\right)}{4}+\frac{\varphi_{\text {in }}}{2}+K_{r} \frac{\cos \left(2 \varphi_{\text {in }}\right)}{4}\right)
\end{aligned}
$$

else,

$$
\begin{aligned}
& F_{x}=0 \\
& F_{y}=0
\end{aligned}
$$

$f_{z}$ is the feed rate and $a_{p}$ is the depth of cut. Then, the global cutting force exerted by the entire tool on the piece can be calculated. Coefficients $K_{t}$ and $K_{r}$ were determined from cutting experiments with a force measurement system. 


\section{IDENTIFICATION OF THE JOINT STIFFNESS VAL- UES}

The translational and rotational displacements of the robot end-effector can be evaluated if the forces and moments applied on it are known. To this end, the stiffness model of the robot defined by Eq. (5) was determined based on the CCT Theory [8]. The links of the robot are supposed to be quite stiffer than the joints and not known as it happens usually for industrial robots [9]. Then, the relationship between the wrench applied on the robot end-effector and its small displacement screw is defined as follows:

$$
\boldsymbol{w}=\mathbf{K}_{X} \delta \boldsymbol{X}
$$

where $\mathbf{K}_{X}$ is the Cartesian stiffness matrix of the robot. $\boldsymbol{w}$ is the 6-dimensional vector of forces and moments exerted on the robot end-effector and derived from Sec. 2. $\delta \boldsymbol{X}$ is the obtained 6-dimensional small displacement screw of the end-effector.

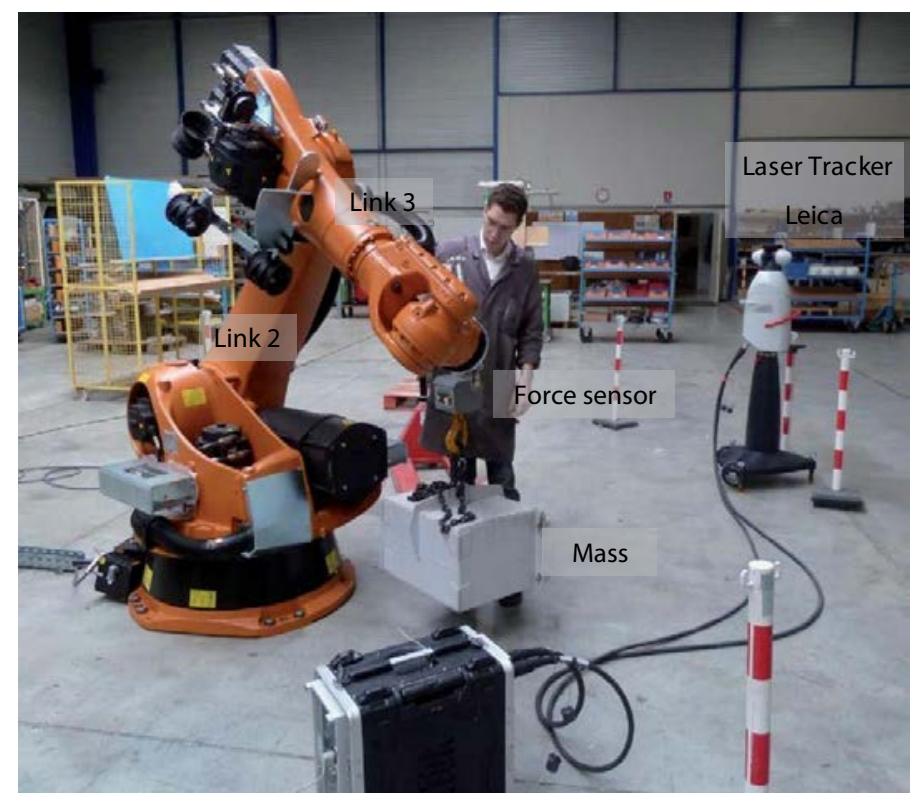

FIGURE 2. Experimental setup for the joint stiffness identification of the KUKA KR270-2 robot

The Cartesian stiffness matrix $\mathbf{K}_{X}$ that depends on the robot configuration, the joint stiffness values and the wrench applied on the end-effector takes the form:

$$
\mathbf{K}_{X}=\mathbf{J}^{-T}\left(\mathbf{K}_{\theta}-\mathbf{K}_{C}\right) \mathbf{J}^{-1}
$$

with

$$
\mathbf{K}_{\theta}=\left[\begin{array}{cccccc}
k_{\theta_{1}} & 0 & 0 & 0 & 0 & 0 \\
0 & k_{\theta_{2}} & 0 & 0 & 0 & 0 \\
0 & 0 & k_{\theta_{3}} & 0 & 0 & 0 \\
0 & 0 & 0 & k_{\theta_{4}} & 0 & 0 \\
0 & 0 & 0 & 0 & k_{\theta_{5}} & 0 \\
0 & 0 & 0 & 0 & 0 & k_{\theta_{6}}
\end{array}\right]
$$

and

$$
\mathbf{K}_{C}=\left[\frac{\partial \mathbf{J}^{T}}{\partial \theta_{1}} \boldsymbol{w} \frac{\partial \mathbf{J}^{T}}{\partial \theta_{2}} \boldsymbol{w} \frac{\partial \mathbf{J}^{T}}{\partial \theta_{3}} \boldsymbol{w} \frac{\partial \mathbf{J}^{T}}{\partial \theta_{4}} \boldsymbol{w} \frac{\partial \mathbf{J}^{T}}{\partial \theta_{5}} \boldsymbol{w} \frac{\partial \mathbf{J}^{T}}{\partial \theta_{6}} \boldsymbol{w}\right]
$$

$\mathbf{J}$ is the kinematic Jacobian matrix of the robot. $\mathbf{K}_{\theta}$ is its diagonal joint stiffness matrix and $\mathbf{K}_{C}$ is the complementary stiffness matrix, which depends on the wrench applied on the end-effector.

TABLE 1. Apparent joint stiffness values in [MNm/rad] of the KUKA KR270-2 robot

\begin{tabular}{cccccc}
\hline \hline$k_{\theta_{1}}$ & $k_{\theta_{2}}$ & $k_{\theta_{3}}$ & $k_{\theta_{4}}$ & $k_{\theta_{5}}$ & $k_{\theta_{6}}$ \\
0.237 & 3.32 & 2.79 & 0.486 & 0.521 & 0.38 \\
\hline \hline
\end{tabular}

An identification procedure was developed in [10] in order to determine all joint stiffness values of the robot. Accordingly, the translational and rotational displacements of the end-effector can be predicted knowing the wrench exerted on it. It is noteworthy that the flexibility of the links is considered through the identified joint stiffness values. The joint stiffness values of the KUKA KR270-2 robot are given in Table 1 . They were obtained by using the experimental setup shown in Fig. 2] and described in [11,12].

\section{INFLUENCE OF THE WORKPIECE PLACEMENT AND THE KINEMATIC REDUNDANCY ON THE MA- CHINING QUALITY}

This section aims to show the influence of the workpiece placement and the kinematic redundancy on the machining quality of a test workpiece through some experimental results.

\subsection{Evaluation of the tool displacement during a milling operation}

Knowing the joint stiffness values of the robot from Sec. 3 and the forces exerted on the robot end-effector from Sec. 2, it is possible to predict the tool displacement using the stiffness matrix defined with Eq. (5). Four machining operations were 
TABLE 2. Cutting conditions

\begin{tabular}{cccc}
\hline \hline Reference & $N[\mathrm{tr} / \mathrm{min}]$ & $a_{p}[\mathrm{~mm}]$ & $V_{f}[\mathrm{~mm} / \mathrm{min}]$ \\
\hline P3 & 20000 & 5 & 3600 \\
P4 & 10000 & 5 & 3600 \\
P5 & 6670 & 5 & 3600 \\
\hline \hline
\end{tabular}

realized in order to validate the theoretical elastostatic model of the KUKA KR270-2 robot: (i) a workpiece made up of a resin material called LAB was milled along a $300 \mathrm{~mm}$ linear path. It is noteworthy that the LAB material exerts a small force on the tool; (ii) a workpiece made up of an Aluminium alloy was milled along a $300 \mathrm{~mm}$ linear path with the cutting conditions $\mathrm{P} 3$ given in Table 2, (iii) a workpiece made up of an Aluminium alloy was milled along a $300 \mathrm{~mm}$ linear path with the cutting conditions $\mathrm{P} 4$ given in Table 2, (iv) a workpiece made up of an Aluminium alloy was milled along a $300 \mathrm{~mm}$ linear path with the cutting conditions $\mathrm{P} 5$ given in Table 2. $N, a_{p}$ and $V_{f}$ denote the rotational speed of the tool, the depth of cut and the feed speed, respectively.

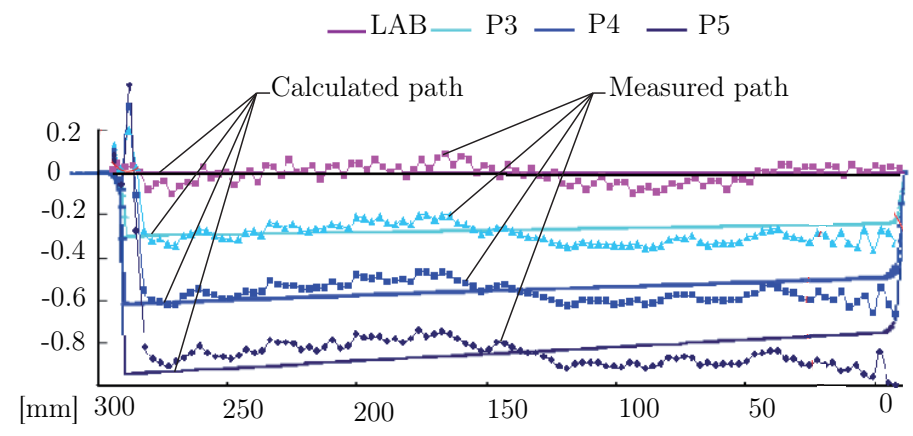

FIGURE 3. Calculated and measured paths associated with four milling conditions

Figure 3 illustrates the corresponding measured and calculated paths. The inherent straightness of the robot explains the difference between the calculated and measured paths in the LAB material. We can notice that the difference between the calculated and measured paths for the other machining operations is about 5\% and is also partly due to the inherent straightness of the robot. Consequently, it is possible to evaluate the path that will be followed by the tool considering its displacement due to the cutting forces from the elastostatic model of the robot defined with Eq. (5).

\subsection{Influence of the task placement on the machining quality}

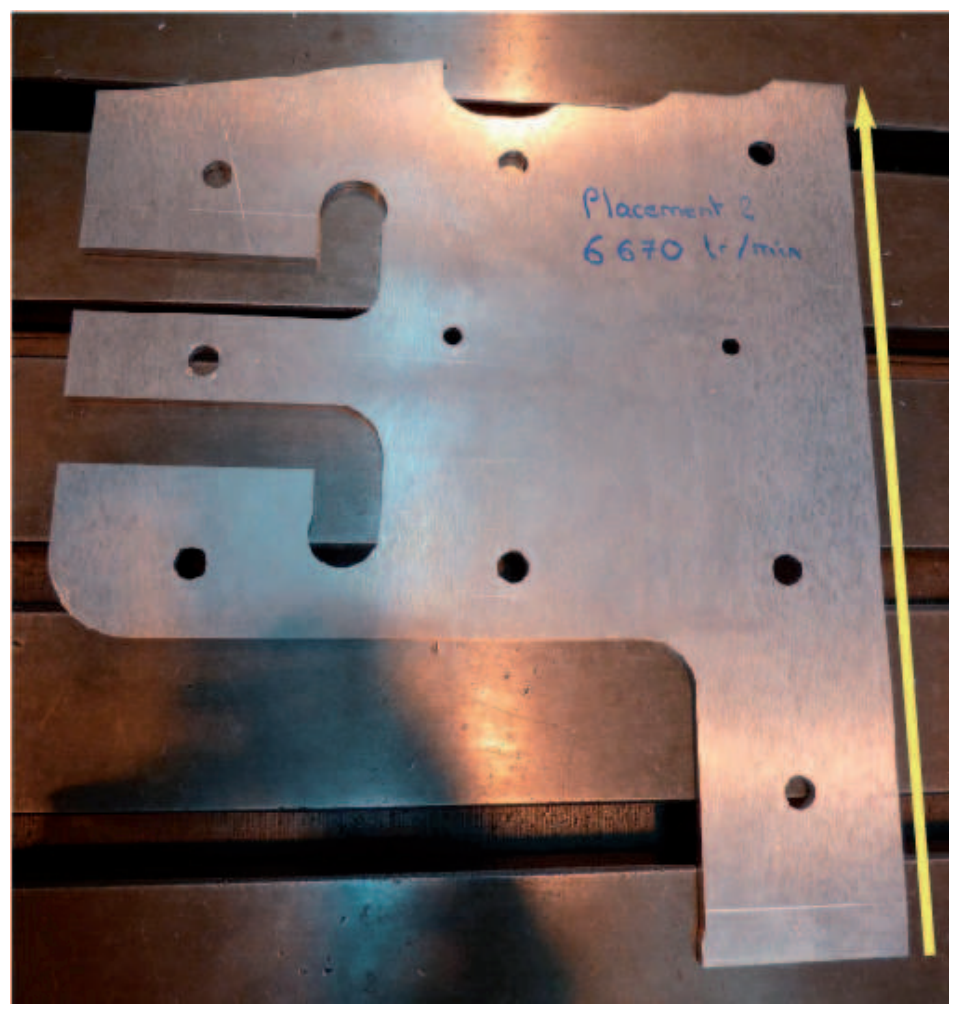

FIGURE 4. Test workpiece

In order to show the influence of the task placement on the machining quality, a test workpiece shown in Fig. 4 was milled in two different placements within the robot Cartesian workspace. The two placements of the test workpiece are illustrated in Fig.5 Four machining operations were realized with the same cutting condition, namely, the cutting condition P4 described in Table 2 . (i) the milling of the test workpiece made up of a LAB material located in Placement 1; (ii) the milling of the test workpiece made up of an Aluminium alloy located in Placement 1; (iii) the milling of the test workpiece made up of a LAB material located in Placement 2; (iv) the milling of the test workpiece made up of an Aluminium alloy located in Placement 2.

Figure 6 shows the measures of the four resulting workpieces, namely, the milling quality of the four resulting workpieces. It is apparent that the resulting workpieces made up of a LAB material are closed to the desired workpiece represented with the black straight line in Fig. 6, while there is a non negligible gap betweeen the resulting workpieces made up of an Aluminium alloy and the desired workpiece. Moreover, the difference between the two resulting workpieces made up of an Alu- 


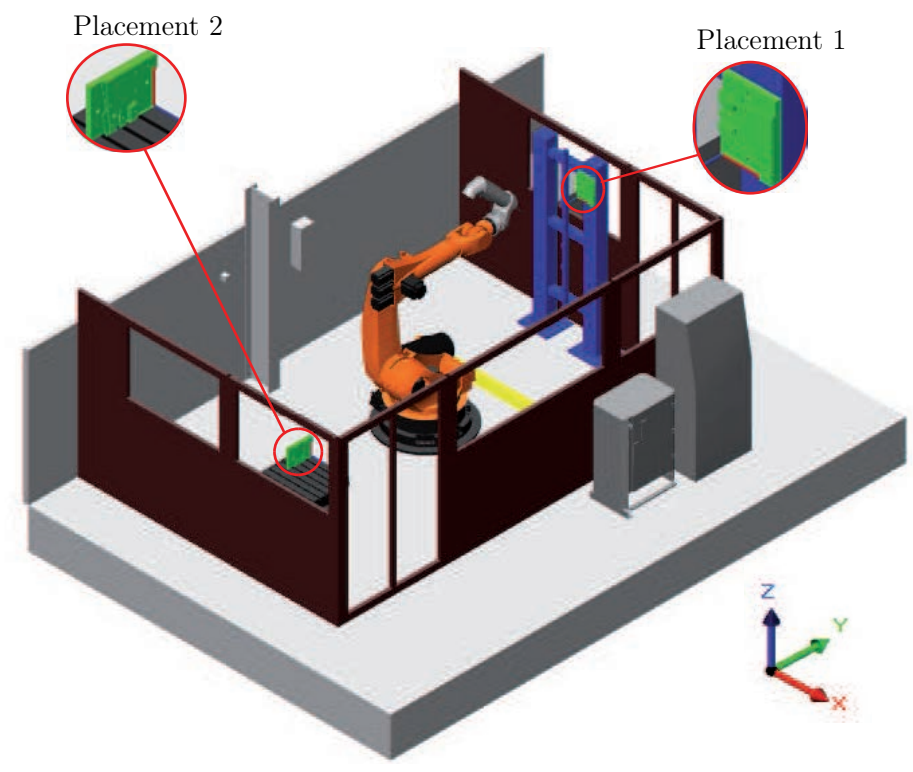

FIGURE 5. Two placements of the test workpiece

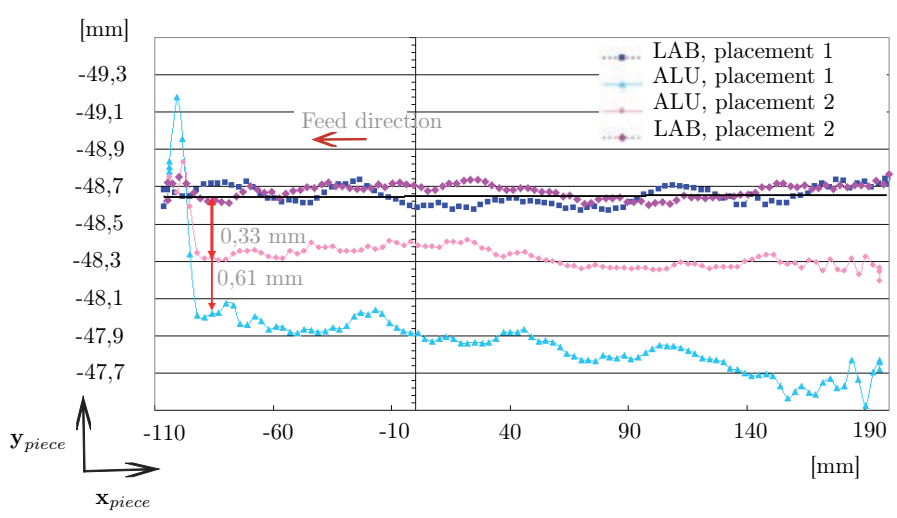

FIGURE 6. Measurement of the test workpiece milled in two different placements

minium alloy is important. As a consequence, we can claim that the placement of the workpiece really affects its milling quality.

\subsection{Influence of the kinematic redundancy on the ma- chining quality}

The KUKA KR270-2 robot has six degrees of freedom. The milling operations sets only five degrees of freedom because the rotation of the spindle about the tool axis is not fixed. Therefore, the robot is redundant with respect to the task, the robot redundancy being equal to one as explained in [14,15]. Here, the robot redundancy is characterized by the angle $\beta$, which denotes the rotation angle of the spindle about the tool axis and is depicted in Fig. 7. Figure 8 illustrates the quality of two test workpieces made up of an aluminium alloy and milled in the same place-

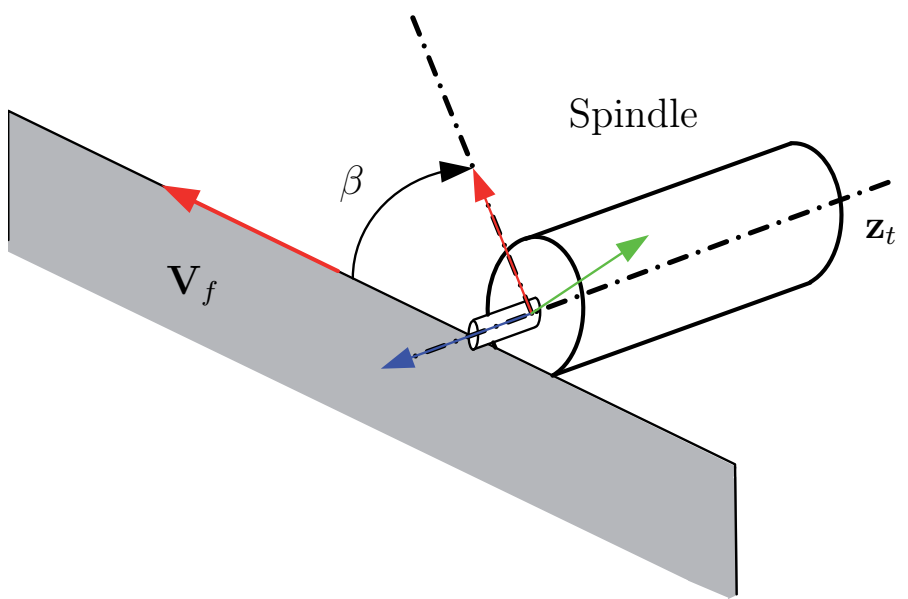

FIGURE 7. Kinematic redundancy characterized with angle $\beta$

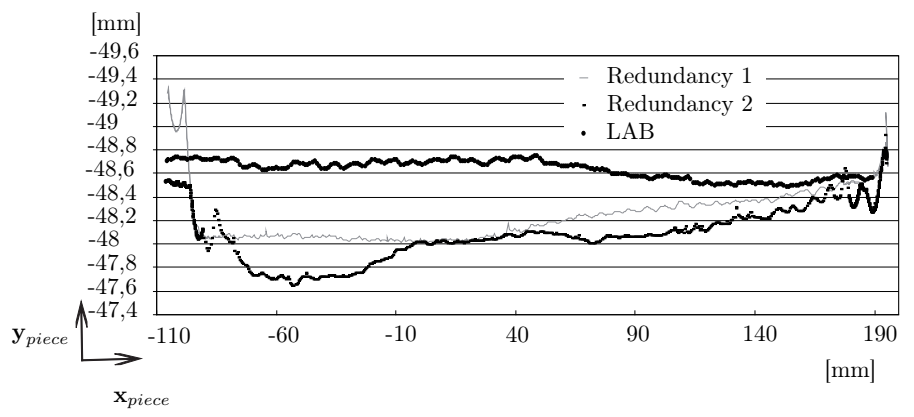

FIGURE 8. Quality of the test workpiece milled in a given placement, but with two different redundancy planning schemes

ment, but with two different redundancy planning schemes. It is apparent that the milling quality also depends on the redundancy planning scheme chosen for the machining operation.

\section{OPTIMUM PLACEMENT OF THE WORKPIECE}

This section presents a methodology that aims to determine the best placement of the workpiece to be machined knowing the cutting forces exerted on the tool and the elastostatic model of the robot. The proposed methodology is explained through the definition of a machining quality criterion and the formulation of a mono-objective optimization problem.

\subsection{Machining quality criterion}

The tool displacement along the normal to both the feed direction and the tool axis is mainly responsible for the machining errors shown in Figs. 3 , 6 and 8 .

Let us discretize the path to be followed during the machining operation into $n$ points. Let $c_{i}$ be the tool displacement along the normal to both the feed direction $\mathbf{V}_{f, i}$ and the tool axis $\mathbf{z}_{t, i}$ 
at the $i$ th point of the discretized path. $c_{i}$ can be expressed as follows:

$$
c_{i}=\delta \mathbf{d}_{i}^{T}\left(\mathbf{V}_{f, i} \times \mathbf{z}_{t, i}\right)
$$

where $\delta \mathbf{d}_{i}$ is the point-displacement of the tool at the $i$ th point obtained from Eq. (5) and $\times$ denotes the cross product.

Therefore, the machining quality criterion $f_{M Q C}$, which is the objective function of the optimization problem at hand is defined as the root mean square of the $c_{i}$ values, namely,

$$
f_{M Q C}=\sqrt{\frac{1}{n}\left(c_{1}^{2}+c_{2}^{2}+\cdots+c_{n}^{2}\right)} \rightarrow \min
$$

The smaller $f_{M Q C}$, the better the machining quality.

\subsection{Decision variables}

The decision variable vector of the optimization problem contains the Cartesian coordinates of the workpiece geometric center and its orientation parameters expressed in the robot base frame. Let $x_{p}, y_{p}$ and $z_{p}$ be the Cartesian coordinates of the workpiece geometric center expressed in the robot base frame along the $x$-, $y$ - and $z$-axes, respectively. The orientation of the workpiece can be fully represented with the variables $\left(Q_{2}, Q_{3}\right.$, $Q_{4}$ ), a subset of the quaternions coordinates. Indeed, the quaternions represent the rotations of the workpiece with a rotation axis $\mathbf{u}=\left[u_{x} u_{y} u_{z}\right]^{T}$ and an angle $\theta$. The relation between the quaternions and the axis and angle representation can be found in [16]:

$$
\begin{aligned}
Q_{1} & =\cos (\theta / 2) \\
Q_{2} & =u_{x} \sin (\theta / 2) \\
Q_{3} & =u_{y} \sin (\theta / 2) \\
Q_{4} & =u_{z} \sin (\theta / 2)
\end{aligned}
$$

where $u_{x}^{2}+u_{y}^{2}+u_{z}^{2}=1$ and $0 \leq \theta \leq \pi$.

The decision variable vector also contains the redundancy planning scheme. Let $\beta_{i}$ be the rotation angle of the spindle about the tool axis, namely, the angle $\beta$ shown in Fig. 7, at the $i$ th point of the discretized path, $i=1, \ldots, n$. Accordingly, the redundancy planning scheme is defined by vector $\boldsymbol{\beta}$ expressed as:

$$
\boldsymbol{\beta}=\left[\begin{array}{llll}
\beta_{1} & \beta_{2} \cdots & \beta_{n}
\end{array}\right]^{T}
$$

The KUKA KR270-2 robot may have eight solutions to the inverse geometric model. Let $\mu$ denote the solution number to the inverse geometric model of the robot. Therefore, $\mu$ is also a decision variable of the optimization problem.

\subsection{Constraints}

Four types of constraints arise in the optimization problem at hand:

1. The joint angles of the KUKA KR270-2 robot should be bounded between its joint limits, namely,

$$
\begin{aligned}
-185 \mathrm{deg} & \leq \theta_{1 i} \leq 185 \mathrm{deg} \\
0 & \leq \theta_{2 i} \leq 146 \mathrm{deg} \\
-245 \mathrm{deg} & \leq \theta_{3 i} \leq 29 \mathrm{deg} \\
-350 \mathrm{deg} & \leq \theta_{4 i} \leq 350 \mathrm{deg} \\
-125 \mathrm{deg} & \leq \theta_{5 i} \leq 125 \mathrm{deg} \\
-350 \mathrm{deg} & \leq \theta_{6 i} \leq 350 \mathrm{deg}
\end{aligned}
$$

$\theta_{j i}$ being the $j$ th joint angle of the robot, $j=1, \ldots, 6$, when the tool is located at the $i$ th point of the discretized path, $i=1, \ldots, n$. Note that the robot was parameterized by using the modified Denavit-Hartenberg convention [16].

2. The tool displacement $c_{i}$ expressed with Eq. (9), $i=1, \ldots, n$, should be smaller than a given value $c_{\max }$, which is defined with regard to the expected machining quality, i.e.,

$$
\max \left(\left|c_{1}\right|,\left|c_{2}\right|, \cdots,\left|c_{n}\right|\right) \leq c_{\max }
$$

where $|$.$| denotes the absolute value.$

3. The workpiece should be located within an area of the robot cell free of obstacles, namely,

$$
\begin{aligned}
& x_{\text {pmin }} \leq x_{p} \leq x_{\text {pmax }} \\
& y_{\text {pmin }} \leq y_{p} \leq y_{\text {pmax }} \\
& z_{\text {pmin }} \leq z_{p} \leq z_{\text {pmax }}
\end{aligned}
$$

where $x_{p \min }, y_{p \min }, z_{\text {mmin }}$ and $x_{p \max }, y_{p \max }, z_{p \max }$ are the lower and upper bounds of the Cartesian coordinates $x_{p}, y_{p}$, $z_{p}$ of the workpiece geometric center, respectively. It is noteworthy that those lower and upper bounds are defined with regard to the obstacles within the robot cell in order to be sure that the workpiece does not collide any obstacle.

4. The robot should not meet any singularity while the tool is following the path, namely,

$$
\left|\operatorname{det}\left(\mathbf{J}_{i}\right)\right|>0, \quad i=1, \ldots, n
$$

$\mathbf{J}_{i}$ being the kinematic Jacobian matrix of the robot defined in [12] and evaluated at the $i$ th point of the path.

5. The robot joint trajectory should be continuous. 


\subsection{Formulation of the optimization problem}

From Eqs. (10) to 26), the optimization problem to be solved to find the best workpiece placement within the robot cell can be formulated as follows:

$$
\begin{aligned}
& \text { minimize } f_{M Q C}
\end{aligned}
$$

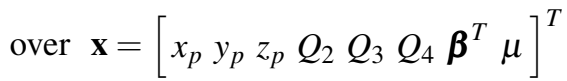

$$
\begin{aligned}
& \text { subject to } \max \left(\left|c_{1}\right|,\left|c_{2}\right|, \cdots,\left|c_{n}\right|\right) \leq c_{\max } \\
& -185 \mathrm{deg} \leq \theta_{1 i} \leq 185 \mathrm{deg} \\
& 0 \leq \theta_{2 i} \leq 146 \mathrm{deg} \\
& -245 \mathrm{deg} \leq \theta_{3 i} \leq 29 \mathrm{deg} \\
& -350 \mathrm{deg} \leq \theta_{4 i} \leq 350 \mathrm{deg} \\
& -125 \mathrm{deg} \leq \theta_{5 i} \leq 125 \mathrm{deg} \\
& -350 \mathrm{deg} \leq \theta_{6 i} \leq 350 \mathrm{deg} \\
& \left|\operatorname{det}\left(\mathbf{J}_{i}\right)\right|>0 \\
& x_{\text {pmin }} \leq x_{p} \leq x_{\text {pmax }} \\
& y_{\text {pmin }} \leq y_{p} \leq y_{\text {pmax }} \\
& z_{\text {pmin }} \leq z_{p} \leq z_{\text {pmax }} \\
& \mu \in[1, \ldots, 8] \\
& i=1, \ldots, n
\end{aligned}
$$

Therefore, optimization problem (27) aims to find the optimum workpiece placement and the corresponding optimum redundancy planning scheme that minimize the machining quality criterion $f_{M Q C}$ defined by Eq. (10) while respecting the set of the constraints expressed by Eqs. (16) to 26).

An illustrative example is presented in the next section in order to explain how optimization problem (27) can be solved.

\section{ILLUSTRATIVE EXAMPLE}

This illustrative example aims to find the optimal placement of the workpiece illustrated in Fig. 9. The 2-norms of the tangential cutting force $\mathbf{F}_{t, i}$ and radial cutting force $\mathbf{F}_{r, i}$ applied by the workpiece on the tool at the $i$ th point are:

$$
\begin{aligned}
& \left\|\mathbf{F}_{t, i}\right\|_{2}=1500 \mathrm{~N} \\
& \left\|\mathbf{F}_{r, i}\right\|_{2}=500 \mathrm{~N}
\end{aligned}
$$

The milling path is a rectangle of size:

$$
\begin{aligned}
w_{W P} & =0.6 \mathrm{~m} \\
h_{W P} & =0.3 \mathrm{~m}
\end{aligned}
$$

Besides, the workpiece is supposed to stay in a horizontal configuration within the robot base frame, i.e.,

$$
Q_{2}=Q_{3}=0
$$

The workpiece is free to rotate about the vertical axis, namely,

$$
-1 \leq Q_{4} \leq 1
$$

Nevertheless, the geometric center of the workpiece is bounded, namely,

$$
\begin{aligned}
x_{\text {pmin }} & =0.4 \mathrm{~m} \\
x_{\text {pmax }} & =1.1 \mathrm{~m} \\
y_{\text {pmin }} & =0.4 \mathrm{~m} \\
y_{\text {pmax }} & =1.1 \mathrm{~m} \\
z_{\text {pmin }} & =0.3 \mathrm{~m} \\
z_{\text {max }} & =1.1 \mathrm{~m}
\end{aligned}
$$

Moreover, the maximum point-dispacement $c_{\max }$ of the tool should be smaller than $3 \mathrm{~mm}$ because of the expected machining quality, i.e.,

$$
c_{\max }=3 \mathrm{~mm}
$$

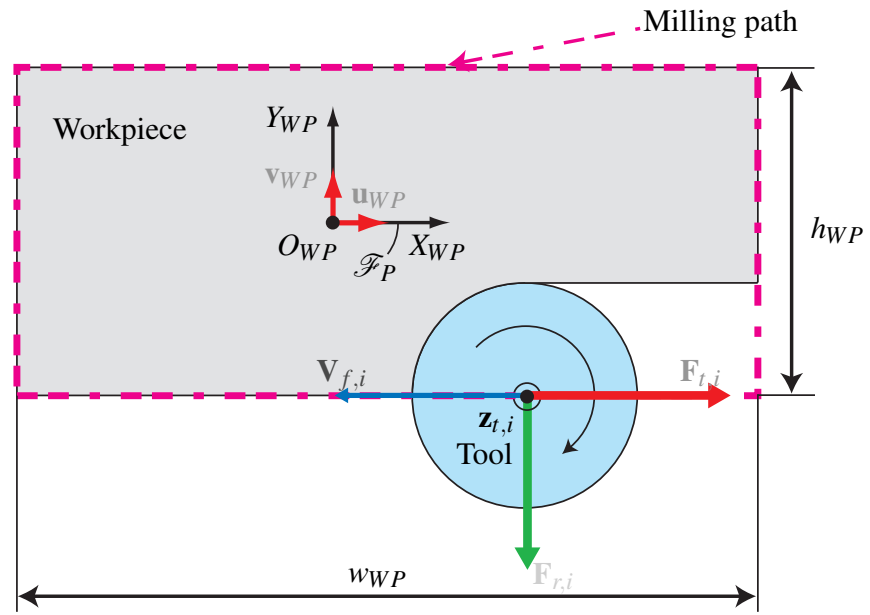

FIGURE 9. Workpiece and cutting forces

A hybrid optimization algorithm was used to solve optimization problem (27) with the foregoing data. As a matter of fact, a genetic algorithm [17] was used to find the initial guess of an interior-point algorithm for large-scale nonlinear programming [18]. In this vein, the Matlab ga function combined with 


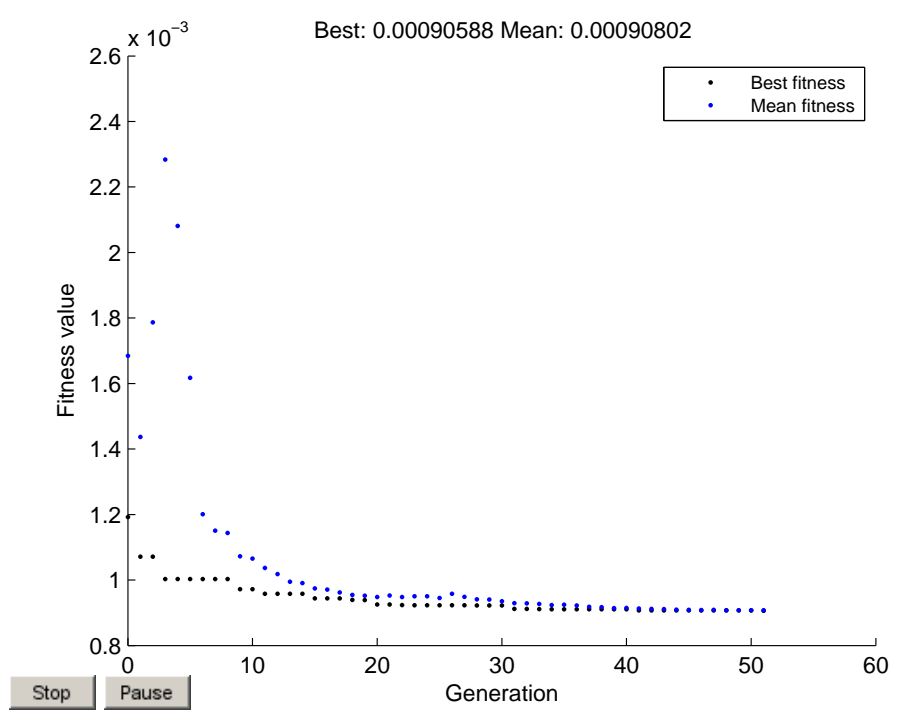

FIGURE 10. Convergence of the genetic algorithm

the Matlab fmincon function were used. Figure 10 illustrates the convergence of the genetic algorithm. The latter converged after 51 generations, each population containing 20 individuals. Then, a local optimum decision variable vector $\mathbf{x}_{\text {opt }}$ of optimization problem 27) was obtained with the Matlab fmincon function and is expressed as:

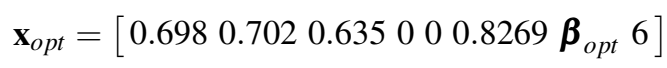

Figure 11 shows the isocontours of $c_{i}$ with respect to the path

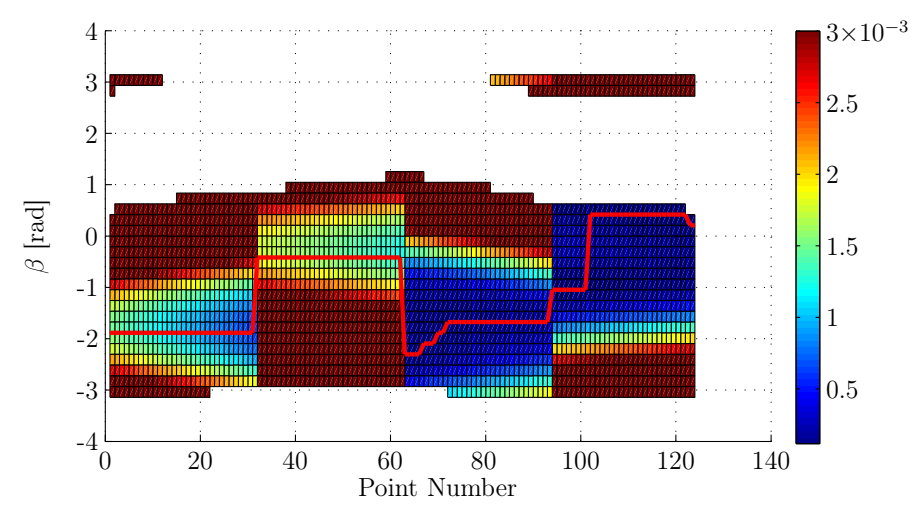

FIGURE 11. Isocontours of $c_{i}$ [m] w.r.t the path point number and $\beta$ angle for the optimum workpiece placement. The red curve characterizes the optimum redundancy planning scheme, namely, the $\boldsymbol{\beta}_{\text {opt }}$ vector

point number and $\beta$ angle for the optimum milling path place- ment. The red curve characterizes the optimum redundancy planning scheme, i.e., the $\boldsymbol{\beta}_{\text {opt }}$ vector. The white areas can not be reached by the robot because of its joint limits expressed by Eqs. (16) to 21). Besides, the point-displacement of the tool is higher than $c_{\text {max }}$ in the red areas.

It is noteworthy that $x_{p}, y_{p}, z_{p}, Q_{2}, Q_{3}, Q_{4}$ and $\mu$ are the only decision variables considered by the $g a$ and fmincon functions in this optimization problem solving. As a matter of fact, an optimal $\boldsymbol{\beta}$ vector is searched at each iteration of the genetic algorithm and at each iteration of the interior-point algorithm. This vector is obtained by minimizing the noint displacement of the tool at each point of the milline

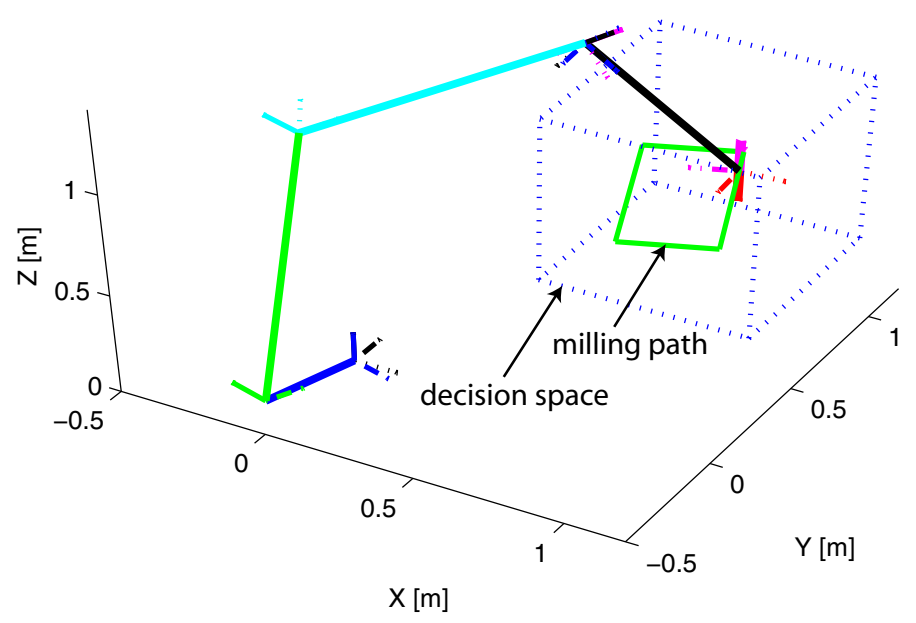

FIGURE 12. Optimum milling path placement

Figure 12 depicts the optimum milling path placement and the decision space defined with Eqs. (34) to (39). An animation of the corresponding optimum milling operation can be downloaded 1

The optimum redundancy planning scheme, i.e., the red curve in Fig. 11, appears to be piecewise continuous. It should be considered during the cutting strategy to avoid signs of wear on the workpiece after the milling operation. As a consequence, a cutting strategy is illustrated in Fig. 13 to avoid signs of wear in the workpiece corners.

\section{CONCLUSIONS}

This paper introduced a methodology to determine the optimum placement of any workpiece to be machined knowing the cutting forces exerted on the tool and the elastostatic model of the KUKA KR270-2 robot. First, a cutting force model was presented to predict the cutting forces applied by the tool on the

http://www.irccyn.ec-nantes.fr/ caro/ASME ESDA2012/OptimSolKR270.avi 
(a)
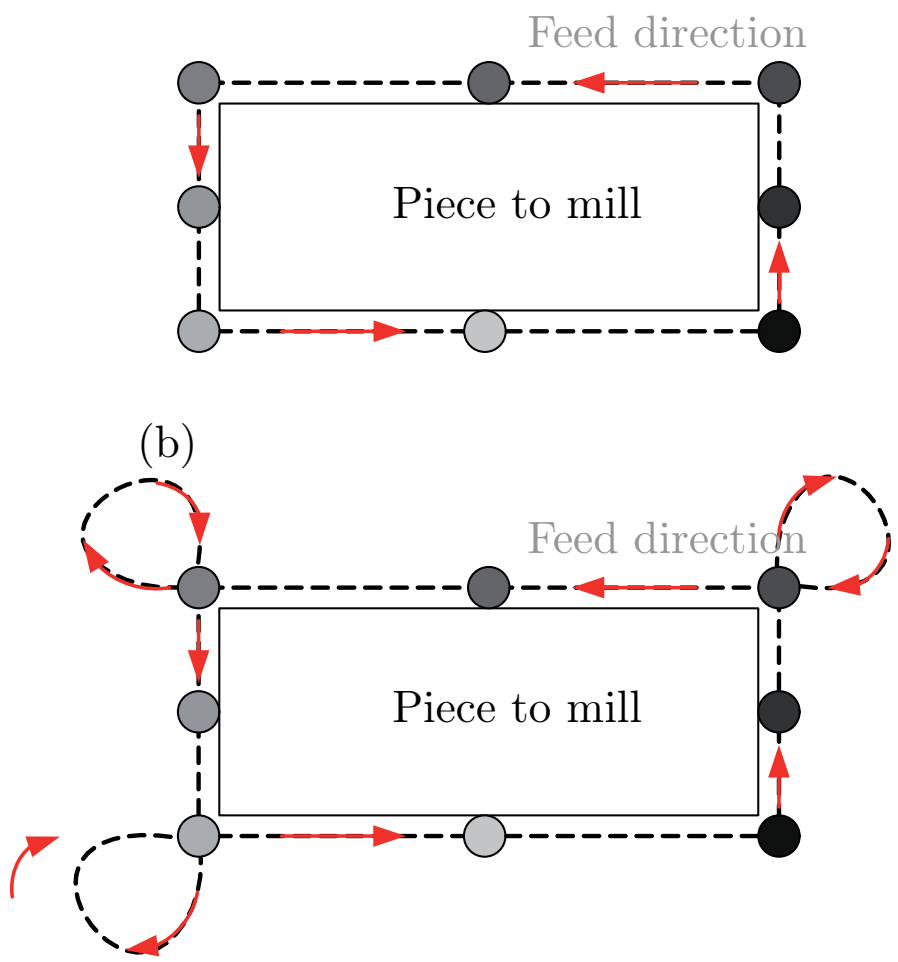

FIGURE 13. Modification of the cutting strategy to avoid signs of wear on the workpiece

workpiece. Then, a joint stiffness identification procedure was explained and the joint stiffness values of the KUKA KR2702 robot were given. The influence of the workpiece placement and the kinematic redundancy on the machining quality of a test workpiece was highlighted through some experimentations. A mono-objective optimization problem was formulated to determine the optimum placement of a workpiece to be machined. Finally, an illustrative example was developed in order to show the contributions of the paper.

The proposed methodology will help the robot user find the optimum placement of any workpiece to be machined within the Cartesian workspace of any six-revolute industrial serial robot. It will also help him/her deal with the kinematic redundancy of the robot with regard to the task.

The experimental validations of the results and the extension of the proposed methology to robot machining with a higher kinematic redundancy of the robot with regard to the task are part of the future works.

\section{ACKNOWLEDGMENT}

The authors would like to acknowledge the financial support of the ANR, France (Project ANR-2010-SEGI-003-02COROUSSO).

\section{REFERENCES}

[1] Terrier, M., Dugas, A., Hascoet, J.-Y. (2005). "Machinesoutils à structure parallèle et usinage à grande vitesse," Mécanique \& Industrie, 6, pp. 431-437.

[2] Matsuoka, S.-I.,Shimizu, K.,Yamazaki, N. and Oki, Y. (1999). "High-Speed End Milling of an Articulated Robot and its Characteristics," Elsevier, Journal of Materials Processing Technology, 95, pp. 83-89.

[3] Zhang, H., Hang, H., Wang, J., Zhang, G., Gan, Z., Pan, Z., Cui, H. and Zhu, Z. (2005). "Machining with Flexible Manipulator: Toward Improving Robotic Machining Performance," Proceedings of the 2005 IEEE/ASME International Conference on Advanced Intelligent Mechatronics, Monterey, California, USA, 24-28 July.

[4] Abele, E., Weigold, M. and Rothenbcher, S. (2007). "Modeling and Identification of an Industrial Robot for Machining Applications," Elsevier, Annals of the CIRP, 56/1/2007.

[5] Olabi, A., Bearee, R., Damak, M. and Gibaru, O. (2009). "Planification de l'avance sur trajectoire pour un robot 6 axes usinant," Colloque National AIP PRIMECA, La Plagne, France.

[6] Pan, Z., Zhang, H., Zhu, Z. and Wang, J. (2006). "Chatter Analysis of Robotic Machining Process," Journal of Materials Processing Technology, 173, pp. 301-309.

[7] Ur-Rehman, R., Caro, S., Chablat, D. and Wenger, P. (2010). "Multiobjective Path Placement Optimization of Parallel Kinematics Machines Based on Energy Consumption, Shaking Forces and Maximum Actuators Torques: Application to the Orthoglide," Mechanism and Machine Theory, 45, pp. 1125-1141.

[8] Chen, S.-F. (2003). "The 6x6 Stiffness Formulation and Transformation of Serial Manipulators via the CCT Theory," IEEE International Conference on Robotics \& Automation, Taiwan.

[9] Dumas, C. (2011). "Développement de méthodes robotisées pour le parachèvement de pièces métalliques et composites," $\mathrm{PhD}$ thesis, University of Nantes.

[10] Dumas, C., Caro, S., Garnier, S. and Furet, B. (2011). "Joint Stiffness Identification of Six revolute Industrial Serial Robots," Robotics and Computer Integrated Manufacturing, 27(4), pp. 881-888.

[11] Dumas, C., Caro, S., Cherif, M., Garnier, S. and Furet, B. (2010). "A Methodology for Joint Stiffness Identification of Serial Robots," The 2010 IEEE/RSJ International Conference on Intelligent Robots and Systems (IROS 2010), Taipei, Taiwan, October 18-22. 
[12] Dumas, C., Caro, S., Cherif, M., Garnier, S. and Furet, B. (2011). "Joint Stiffness Identification of Industrial Serial Robots," Robotica, Available on CJO 2011 doi:10.1017/S0263574711000932.

[13] Tlusty, J. and Macneil, P. (1975). "Dynamics of cutting forces in end milling," Annals of the CIRP, 24(1), pp. 2125.

[14] Conkur, E.S. and Buckingham, R. (1997). "Clarifying the definition of redundancy as used in robotics," Robotica, 15, pp. 583-586..

[15] Wenger, P. (2004). "Curve-following for redundant manipulators with obstacles : feasibility analysis and solutions," Journal IFToMM Problems of Applied Mechanics, 24(1), No.2, pp. 17-26.

[16] Khalil, W. and Dombre, E. (2002). Modeling, Identification and Control of Robots, Hermes Penton Ltd.

[17] Goldberg, D.E. (1989). Genetic Algorithms in Search, Optimization \& Machine Learning, Addison-Wesley.

[18] Byrd, R.H., Mary, E.H. and Nocedal, J (1999). "An Interior Point Algorithm for Large-Scale Nonlinear Programming," SIAM Journal on Optimization, 9(4), pp. 877-900. 